\title{
Subjective pictorial information and visual search'
}

\author{
IRWIN POLLACK AND DAVID SPENCE \\ MENTAL HEALTH RESEARCH INSTITUTE, UNIVERSITY OF MICHIGAN
}

Color pictures were subdivided into $701 \frac{11 / 4}{4}$ square "jig. saw" sections. The individual sections were rated by observers in terms of their apparent informational contribution to the entire picture. The concept of apparent information must have some basis in our culture because large differences in ratings were assigned to the sections. The sections were then employed in visual search tests. Errors and search times tended to be lowest for pictorial segments of the highest-rated apparent information.

"Thou must know thy stimulus" is the Eleventh Commandment of the sensory psychophysicist. By insisting on the adherence to this commandment, sensory psychophysicists have carved an important place in the scientific study of human behavior. In the study of form perception, the admonition to know thy stimulus can be rigorously followed. For example, the method of Attneave and Arnoult (1956) economically specifies classes of shapes, usually nonsense figures with a varying number of sides, by describing the rules for generating the shapes. A brief, but detailed review and discussion of the Attneave-Arnoult approach may be found in Graham (1965). The Attneave-Arnoult rules can be well specified so that high speed digital computers can generate a sufficient number of shapes to satisfy even the most voracious investigator (Brown, Condon, \& Hitchcock, 1966).

With such methodological power available for the study of form perception, why should investigators look elsewhere? Investigators interested in the study of the perception of real visual materials are impatient with restrictions to random shapes. If the real visual world is complicated, so let it be. If compromises are to be made, such investigators would rather compromise in terms of depth of specification than upon subject matter. We interpret Gibson (1966) to be sympathetic to this position. But, until recently, there was not a satisfactory quantitative experimental approach to the study of real pictures.

Norman Mackworth and his associates (Mackworth; Mackworth \& Bruner; Mackworth \& Morandi; Schissler \& Mackworth) have recently provided an experimental approach to the study of the perception of real pictures. Through the use of eye movement recordings, subjective ratings of informational content, and performance tasks with real pictures, they have sought to identify the specific details of real pictures which provide the information bearing elements of real pictures to the observer. We became so fascinated with the potential of this approach for the study of real pictures that we sought to replicate one feature of the Mackworth program: the relation between rated information and the performance upon visual search tests. The approach was to specify elementary sections of a picture in terms of ratings of informational content and to test whether items of high rated information fare better than items of low rated information in a variety of search tests. Because of the small number of pictures employed and because of our lack of correlative eye movement records, we have made no serious attempt to specify why certain sections of pictures are more informative to the picture than are others. Casual inspection reveals the obvious: Sharp contours are associated with high ratings and uniform color masses are associated with low ratings.

\section{MATERIALS}

Five full-page color pictures were obtained from popular magazines. Although we searched many magazines, the ultimate criterion for selection was the availability of two large copies. The selected scenes were: (1) an aerial view of the Pope's visit to St. Patrick's Cathedral (Life, 1965, 59, p. 43); (2) an autumn balcony scene (Better Homes and Gardens); (3) a landscape of Angel Falls, Africa (Life, 1965, 59, p. 80); (4) an explosion over a landscape (Life, 1965, 59, p. 12); and (5) a profile of the face of Mrs. Jacqueline Kennedy (Life, 1965, 59, p. 48).

Each picture was trimmed to $9 \times 12-3 / 4$ in., drymounted, and cut into $1-1 / 4$ in. sections. This size was judged to be a reasonable compromise between tiny colored mosaics with no pictorial information and large heterogeneous sections of both high and low pictorial information.

Each picture was presented to 22 university students on two days separated by a one-week interval. The task of $S$ was to rate the informational contribution of each section.

The specific instructions were: "We are interested in how people assess information in parts of pictures. We would like your help in judging what you feel is the contribution of individual pieces of the entire picture. Stated differently, to what extent would the information of the picture be altered if we removed individual pieces from the picture?"

The $\mathrm{S}$ first identified the pieces with the least and with the most pictorial information and then rated the other pieces on a 12-point rating scale. 


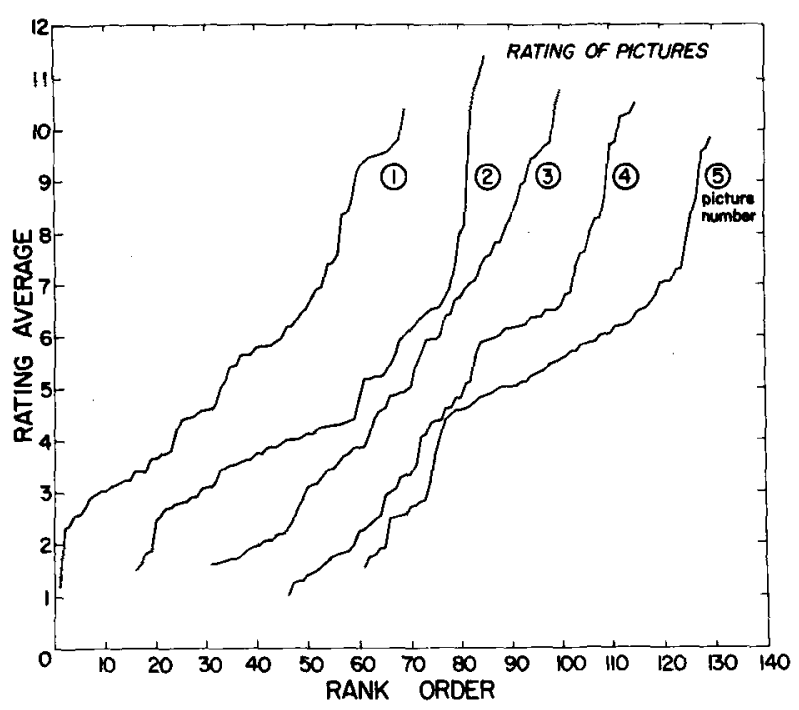

Fig. 1. Average rating scores for $\mathbf{7 0}$ rank-ordered sections of each of five pictures (see text). Successive distributions are shifted by 15 units to the right on the abscissa.

The distribution of ratings for the flve pictures, listed above, is presented in Fig. 1, averaged over Ss and over days. The fact that extreme ratings were obtained suggests that Ss tend to agree closely in rating the informational contribution of portions of pictures, at least for the extreme cases.

The first three pictures listed above were selected for further study on the basis of the distribution of ratings and upon specific local factors, e.g., the lettering on the reverse side of the picture of Mrs. Kennedy was detected through the white background. The 70 sections of each picture were divided into seven sets of 10 each. From one copy of the picture, the first, third, fifth, and seventh sets were chosen as target sections for later tests. Table 1 presents the upper limit, the median, and the lower limit of the ratings (on a scale from 1 to 12) of the 10 sections in each of the four ranks for each picture employed. We shall henceforth refer only to the rank designation of the sections. Rank 1 will refer to the 10 sections of the highest information ratings and Rank 4 will refer to the 10 sections of the lowest information ratings. When only five sections were selected from each set of 10, their average rank was nearly identical to the average rank of the set of 10 .

\section{Precedure}

\section{VISUAL SEARCH TESTS}

In visual search tests, one of the pictorial sections was selected at random and presented to $S$. S's task was to find that piece within the entire array of 70 sections which matched the test section. Three search procedures were employed: HOLD, where $S$ lifted the test section and moved it along the array of 70 pieces; LOOK, where the test section was located in the upper right corner of the frame and $S$ made visual comparisons against the array; and MEMORIZE, where the test section was first presented for $5 \mathrm{sec}$ and then taken away before $S$ searched the array. Search time was monitored by a timer connected to the light in a viewing box. If an error was made, the trial was reinitiated.

In one experiment, nine Ss examined the array of 70 sections arranged in their original order within the picture. In another experiment, nine other Ss examined the array of 70 sections scrambled in a haphazard order within the 7 by 10 array. In all cases, the geographical orientation of each section was maintained in its original direction.

In each experiment, each of the three pictures was presented to $S$ under one of the three search procedures. The order of pictures and procedures was counterbalanced over Ss. Forty of the 70 possible sections -10 in each of the four groups of rated information-served as test sections in search tests with the array in the original order; 20 of the 70 possible sections-five in each of the four groups of rated information-served with the array in scattered order.

\section{Resulis}

The results of the visual search tests are presented in Fig. 2. The abscissa is the rated information of the sections, where 1 refers to sections with the highest rating. The left side of the graph is concerned with error data. The ordinate is the percentage of test sections in which errors were obtained averaged over $\mathrm{Ss}$ and pictures.

Two points are suggested by the data. First, visual search errors are related to rated information. The relationship appears to be more uniform in the case of search over the originally-ordered array than search of the scattered array. This result is reasonable since the pictorial sections were rated within the context of the originally-ordered array.

Second, search errors are related to the remoteness of the test piece. The direct, side-by-side comparisons of the HOLD procedure yielded the lowest error scores and the remote comparison

Table 1. Upper limit, median, and lower limit of ratings of the selected sections.

\begin{tabular}{lrrrr} 
& \multicolumn{5}{c}{ Rank } & 3 & 4 \\
\hline Sicture & 1 & 2 & 4.6 & 3.1 \\
& 10.4 & 6.6 & 4.4 & 2.6 \\
Bolcony Seene & 9.6 & 6.1 & 3.7 & 1.2 \\
& 9.3 & 5.8 & 3.9 & 2.8 \\
Angel Falls & 11.4 & 5.2 & 3.7 & 2.5 \\
& 8.1 & 5.0 & 3.5 & 1.5 \\
& 6.6 & 4.3 & 3.8 & 2.1 \\
& 10.8 & 6.7 & 3.4 & 1.9 \\
& 9.4 & 6.0 & 3.1 & 1.6 \\
\hline
\end{tabular}


by the MEMORIZE procedure yielded the largest error scores. We find puzzling the larger number of errors with the originally-ordered array than with the scattered array under the MEMORIZE procedure with pieces of lowest rated information. We suspect that these pieces are poorly distinguishable from their neighbors when embedded in their original array. But, in the scattered array, sharp boundaries are introduced between neighboring pieces which may assist in isolating the relevant visual features of the lowest rated information sections.

The search times for the correct responses are plotted on the right side of Fig. 2. The times for the memory tests do not include the initial $5 \mathrm{sec}$ inspection. With the exception of the items of lowest ranked information in the scattered array, search time is uniformly shorter for the sections of highest rated information.

\section{COMMUNICATION GUIDANCE}

\section{Procedure}

$S$ did not view the test piece directly. Rather, he listened to another $\mathrm{S}$ describe the piece and attempted to locate the matching piece under one of two conditions: guided or DIRECTED SEARCH while listening to the talker; or listening to the talker's description for $30 \mathrm{sec}$ without visual access to the display, and then searching the display with no guidance other than MEMORY. Two-way communication was not permitted. Each pair of Ss employed one picture under each of the two conditions. Eight pairs of Ss were employed with the array in the original order; eight other pairs were employed with the array in a scattered order. The assignment of pictures and procedures was counterbalanced over each of the two procedures for the first six pairs of Ss. Five pieces were selected from each rank.

\section{Results}

The results for the communication guided search tests are presented in Fig. 3. The general organiza-

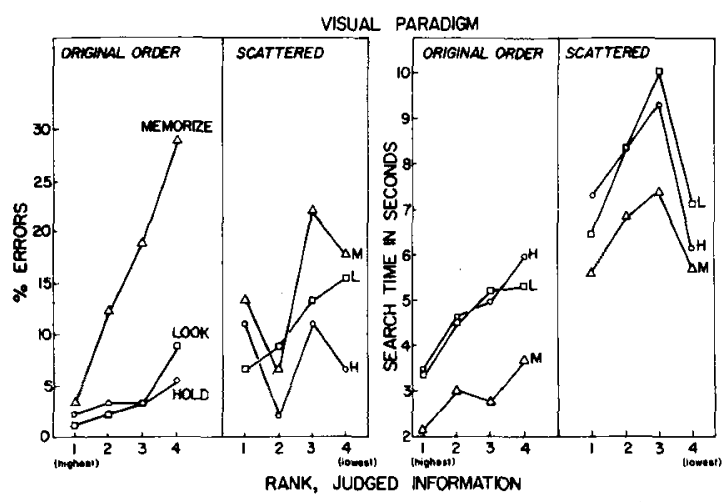

Fig. 2. Percentage error (left section) and average search time (right section) as a function of rated information under three visual procedures.

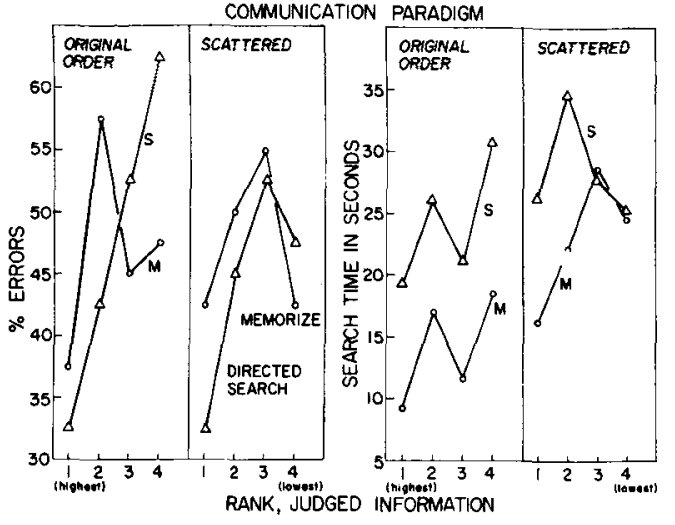

Fig. 3. Percentage error (left section) and average search time (right section) as a function of rated information under two auditory procedures.

tion of Fig. 3 is identical with that of Fig. 2. The initial presentation time with the memorization procedure is again not included. The role of ranked information is somewhat less consistent than with the visual paradigm. In general, however, sections with the highest judged information (Rank 1) were associated with the lowest percentage of errors and with the fastest search times. As in the visual search tests, the pieces with the lowest judged information tended to benefit from a scrambled display format.

\section{DISCUSSION}

The concept of the apparent information of real pictures is supported by two experimental operations:

(1) large differences in ratings among sections of the picture;

(2) correlation of such ratings with speed of search.

As with most exploratory studies, this one raises more questions than it answers. One important question relates to the optimal size of the "piece" in sectioning the picture. Presumably there is no optimal size which is satisfactory for all pictures. Another relates to a possible serious artifact in the method. It may be that the rating procedure forces attention to small detail, which, outside of the experimental situation, $S$ may pass over rapidly. Our impression is that $S$ did not become overwhelmed with small details unless crucial for the meaning of the picture, e.g., an aerial view of the Pope standing on the front steps of St. Patrick's Cathedral. Our final comment has little to do with science, but much to do with the pleasures of experimentation with materials of this type. Ss found the task to be fun!

\section{References}

Attneave, F., \& Amoult, M. D. The quantitative study of shape and pattern perception. Psychol. Bull., 1956, 53, 452-471. 
Brown, D. R., Condon, C. F., \& Hitchcock, L., Jr. Stimulus equivalence of auditory and visual pattems in an intermodal discrimination task. Percept. mot. Skills, 1966, 22, 823-832.

Gibson, J. J. The senses considered as perceptual systems. New York: Houghton Mifflin, 1966, esp. pp. 238-242.

Graham, C. H. Visual form perception. In C. H. Graham (Ed.), Vision and visual perception. New York: Wiley, 1965. Chap. 19, 548-574.

Mackworth, N. H. A stand-mounted eye-marker camera for line-ofsight recording. Percept. \& Psychophys., 1967, 2, 119-127.

Mackworth, N. H., \& Bruner, J. S. Selecting visual information during recognition by adults and children. (In preparation.)
Mackworth, N. H., \& Morandi, A. J. Visual and verbal responses to pictorial stimuli: perceptual differentiation and redundancy effects. Percept. \& Psychophys., in press.

Schissler, D. R., \& Mackworth, N. H. Analysis of pictures by subjective ratings and eye fixations. (In preparation.)

\section{Note}

1. This research was carried out in part under National Science Foundation Grant GB 2894. The author wishes to acknowledge the assistance of Mrs. Linda Silverman and Miss Shelley Barak in constructing the experimental materials.

(Accepted for publication September 12, 1967.) 